\title{
Biological effects of non-ionizing electromagnetic fields at 27 GHz on sperm quality of Mytilus galloprovincialis
}

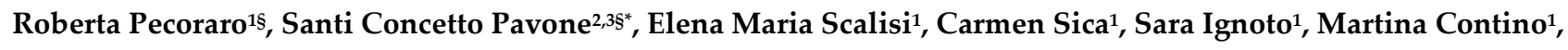 \\ Antonio Salvaggio ${ }^{4}$, Gino Sorbello ${ }^{2,3}$, Loreto Di Donato ${ }^{2,3 \S \$}$ and Maria Violetta Brundo $1 \$ \$^{*}$ \\ 1 Department of Biological, Geological and Environmental Science, University of Catania, Via Androne 81, \\ 95124, Catania, Italy, roberta.pecoraro@unict.it, elena.scalisi@phd.unict.it, carmensica93@gmail.com, \\ sara.ignoto@phd.unict.it, martina.contino@phd.unict.it, mariavioletta.brundo@unict.it \\ 2 Department of Electrical, Electronics, and Computer Engineering (DIEEI), University of Catania, Viale \\ Andrea Doria 6, 95125 Catania, Italy, santi.pavone@unict.it, gino.sorbello@unict.it, loreto.didonato@unict.it \\ 3 Consorzio Nazionale Interuniversitario per le Telecomunicazioni (CNIT), Viale G.P. Usberti, 181/A Pal. 3, \\ 43124 Parma, Italy, santi.pavone@unict.it, gino.sorbello@unict.it, loreto.didonato@unict.it \\ 4 Experimental Zooprophylactic Institute of Sicily “A. Mirri”, Via Gino Marinuzzi, 3, Palermo 90129, Italy, \\ antonio.salvaggio@izssicilia.it \\ $\S$ These authors have contributed equally to this work and share first authorship \\ ss. These authors have contributed equally to this work and share last authorship \\ * Correspondence: mariavioletta.brundo@unict.it; roberta.pecoraro@unict.it Tel.: +390957306039
}

\begin{abstract}
Recently, a rising use of wireless internet technologies has been demonstrated. The devices which use these technologies emit in new spectral regions an electromagnetic radiation (EMFs) which could interact with the male reproductive system. The aim of this study was to investigate in vitro influence of electromagnetic fields at $27 \mathrm{GHz}$ on sperm quality in Mytilus galloprovincialis. Sperm samples, were collected from sexually mature males of $M$. galloprovincialis and placed in seawater. Once evaluated the number and quality of spermatozoa, sperm cells were exposed to electromagnetic fields radiated by a pyramidal horn antenna. The effect of exposure was evaluated after 10, 20, 30,40 and 60 minutes with light microscope and using Eosin test. Ten replications were performed and statistical analysis was carried out by one-way ANOVA test. A significative decrease in sperm motility was observed after 10 minutes of exposure and after 30 minutes most of sperms were immobile and not vital. This study provides useful data on potential ecological impact of the $5 \mathrm{G}$ high-band on animal fertility, whose effect is currently under investigation.
\end{abstract}

Keywords: Mussel; vitality; motility; millimeter waves; SAR

\section{Introduction}

With the roll-out of 5G mobile networks, significantly higher mobile broadband speeds and increasingly wider use of mobile data will be ensured. This is made possible also by the use of additional higher frequency bands [1]. 5G aims to greatly enhance the potentiality of communications, from virtual reality to autonomous vehicles to the industrial internet and smart cities. Furthermore, 5G is considered to be the core technology for the Internet of Things (IoT), where machines communicate with machines [1]. At the same time, a change in exposure to electromagnetic fields (EMF) of humans and the environment is expected. 5G networks will work in different frequency bands and are divided into two different groups [2]. The first group, called Frequency Range 1 (FR1), includes the frequency bands below $6 \mathrm{GHz}$, some of which have already been used by previous standards but extended to cover new portions of the spectrum between $410 \mathrm{MHz}$ and $7125 \mathrm{MHz}$. The second group, called Frequency Range 2 (FR2),includes the frequency range between $24.25 \mathrm{GHz}$ and $52.6 \mathrm{GHz}$ (millimeter waves or mmWave) and has a lower range but allows a wider available bandwidth than the bands of the FR1 group. The 
introduction of this new technology that operates in different frequency bands has attracted a significant amount of toxicity studies [3-5]. To date, however, only a few studies have been conducted on the high frequencies that will be used by 5G [2] and the data are not sufficient to conclude on the existence or not of health effects related to exposure to electromagnetic fields in the band of frequencies around $26 \mathrm{GHz}$ [6]. Tissue heating is the main mechanism of interaction between radiofrequency fields and the human body. The levels of radiofrequency exposure of current technologies cause a negligible increase in temperature in the human body. As the frequency increases, there is less penetration into the body's tissues and the absorption of energy becomes more limited to the surface of the body (skin and eyes). As long as the overall exposure remains below levels fixed by international guidelines, there are no consequences for public health [7]. WHO is conducting a health risk assessment from radio frequency exposure, covering the full range of radio frequencies, including $5 \mathrm{G}$, reviewing literature data on potential health risks from exposure to 5G. Unfortunately, little is known about the effect this new technology could have on coastal marine species. Due to increasing pressure on the environment by humans, biodiversity loss has become one of the greatest environmental concerns. Habitat destruction and overexploitation represent the greatest stressors to marine biodiversity, but excessive anthropization, including the installation of antennas or of repeaters, can also be a threat especially for reproduction of many species [8]. Consequently, artificial electromagnetic fields could impact on the ecological processes in sensitive species, such as spawning or feeding migrations, homing, predation and detection of sexual mates [9-11]. In particular, aquatic invertebrates seems to be sensitive to external factors, and their gametes may be involved at different levels of biological organization [12]. It has been observed an alteration on release of gametes (both spermatozoa and eggs) in seawater, crucial aspect which decreases the reproductive success for the survival of species. Even if some parameters identified as targets of environmental stress are useful biomarkers for the evaluation of exposure to conventional pollutants (pesticides, heavy metals and chemicals substances) [13-16], the effects of electromagnetic fields have not yet been studied. Some evidences suggest that the global environment conditions are changing rapidly and contaminated conditions could interfere with reproductive mechanisms. In this view, some literature studies have shown biological effect on reproductivity at different frequencies band below $6 \mathrm{GHz}[2,5]$, in this paper we have investigated the effect of electromagnetic fields at $27 \mathrm{GHz}$ on sperm quality of bivalve mollusk Mytilus galloprovincialis by in vitro assays. The experiments were conducted with a commercial pyramidal horn antenna with an incident density power not exceeding the international limits stated by the ICNIRP for frequency above $6 \mathrm{GHz}$; spermatozoa were exposed to electromagnetic fields up to 1 hour and during the exposure we have evaluated motility and vitality, important parameters that reflect the health status of the spermatozoa and consequently the reproductive success of species.

\section{Materials and Methods}

\subsection{Exposure Setup Description}

The experiments at $27 \mathrm{GHz}$ were conducted by using a commercial pyramidal horn antenna by XiBao Electronic Tech (model XB-HA28-20) at $27 \mathrm{GHz}$. The dimension of the antenna aperture are $4.4 \times 3.51 \mathrm{~cm}$ and the maximum gain reported in antenna datasheet is $19.23 \mathrm{~dB}$ at $26.50 \mathrm{GHz}$. The antenna is fed by a RF signal generation (R\&S SMB100A) with an output power of $+23 \mathrm{dBm}$ that reduces to $+20 \mathrm{dBm}$ due to insertion loss of the coaxial cable linking RF generator and antenna. The distance between the antenna aperture plane and the 6-well microplates has been fixed to $15 \mathrm{~cm}$ as trade-off between maximum available output power of the RF signal generator and the need to ensure an incident power density comparable with the ICNIRP international guidelines restrictions, that is comparable or, anyway, not exceeding $10 \mathrm{~mW} / \mathrm{cm}^{2}$ [7]. 


\subsection{Experimental Procedure}

Mussels (Mytilus galloprovincialis) were purchased from a mussel farm in Sicily, placed in plastic bags with sea water and immediately transported to the Catania University's Laboratories. We discarded the individuals with obvious signs of breakage of the shell from the sample. Once verified their maturity, the mussels were cleaned from debris and epibionts by manual scraping of the shell, rinsed quickly with water and stabilized for 1 hour before experiments. A number of 20 individuals were selected to induce spawning eggs and sperm by applying a protocol of thermal stimulation (heat shock). Mussels were placed at temperature of $4^{\circ} \mathrm{C}$ for 3-4 hours and then transferred into a tank containing water heated at $28^{\circ} \mathrm{C}$. The water used for the stimulation had a salinity of $30 \pm 1 \%$ and $\mathrm{pH}$ 8.3 and it had been filtered with a $0.22 \mu \mathrm{m}$ filter. After thirty minutes most of the specimens had opened the valve and resumed filtration and they were transferred to a second tank containing sea water at a temperature of $18^{\circ} \mathrm{C}$. Once initiated the spawning, the specimens were immediately removed and placed into an aquarium. Spermatozoa were placed in 6well microplates in $5 \mathrm{ml}$ seawater/well. Control samples (negative control) were incubated only with seawater. The effect of exposure was evaluated up to $1 \mathrm{~h}$ (with observations intervals at 10, 20, 30, 40, 60 minutes). A controlled room temperature has allowed to maintain $22 \pm 1^{\circ} \mathrm{C}$ in wells. Ten replications were performed.

\subsection{Motility analysis}

We measured the motility dividing spermatozoa into two categories: motility and no motility. The analysis was made by placing $10 \mu \mathrm{l}$ of sperms sample on a slide and observing under an optical microscope at x400 magnification. We counted 100 spermatozoa at least.

\subsection{Vitality analysis}

The procedure involves the positioning of $10 \mu \mathrm{l}$ of sperms sample on a slide in which we added $10 \mu \mathrm{l}$ of Eosin $Y(0.5 \%$, Bio-Optica). The observations were made under an optical microscope (Leica DMLB) at x400 magnification. At least 100 spermatozoa were counted. Dead spermatozoa appeared in pink due to the loss of membrane integrity, compared to live spermatozoa that maintain their original coloring.

\subsection{Statistical analyses}

The data obtained were processed for statistical analysis. The vitality and mortality rates between spermatozoa exposed to the electromagnetic fields and the control, were compared using the one-way ANOVA test $(\mathrm{p}<0.001)$ followed by the Tukey test $(\alpha<$ 0.001) using the statistical software Past4Project (version Past4.03). Bar chart graphs of mortality and vitality rate were realized using GraphPad Prism software (version 9.3.1).

\section{Results}

\subsection{Numerical dosimetry analyses}

To establish electromagnetic exposure conditions, numerical simulation has been performed by means of the commercial software CST Microwave Studio. In particular, in the CAD model we have considered the 6-well microplates, the horn antenna and a metallic plane behind the sample holder to take into account agitator for the oxygenation of samples placed at $1 \mathrm{~cm}$ below the 6-well microplates (Fig. 1A). In the experimental setup a polystyrene foam slab has been inserted between the metallic plane and the sample holder to prevent reflecting plane to be very close to aqueous samples, however it has not been considered in the simulation as dielectric parameters of foam can be neglected. The 
dielectric parameters of the materials adopted for the dosimetric analysis at working frequency of $27 \mathrm{GHz}$ are reported in Table 1.

Table 1. Dielectric parameters adopted for the numerical dosimetry.

\begin{tabular}{|c|c|c|c|c|}
\hline Component & Material & $\begin{array}{c}\text { Dielectric } \\
\text { Constant }\end{array}$ & Loss Tangent & Mass density \\
\hline Horn antenna & $\begin{array}{r}\text { Perfect electric conductor } \\
\text { (good metal) }\end{array}$ & - & $\infty$ & - \\
\hline Aqueous sample & Salt water (30\% salinity) & 23.64 & 1.27 & $1029\left[\mathrm{Kg} / \mathrm{m}^{3}\right]$ \\
\hline 6-weel microplates & Polystyrene & 2.5 & 0 & - \\
\hline Ground plane & $\begin{array}{c}\text { Perfect electric conductor } \\
\text { (good metal) }\end{array}$ & - & $\infty$ & - \\
\hline
\end{tabular}

In order to evaluate exposure condition, three complementary metrics have been considered [7]:

- density power of the incident field $\left[\mathrm{W} / \mathrm{m}^{2}\right]$;

- the local specific absorption rate (point SAR) $[\mathrm{W} / \mathrm{Kg}]$;

- power loss density (PLD) deposited into the exposed aqueous samples [W/m³].

The resulting incident power density is reported in Figure $1 \mathrm{~B}$, as it can be seen, although the power density is not uniform above the 6-well microplates, since far field condition are not satisfied [7], it reaches values ranging from 30 to $100 \mathrm{~W} / \mathrm{m}^{2}$ over the antenna footprint area. Taking into account that the metallic plate of the agitator gives rise to a total reflection, these values are comparable with $10 \mathrm{~mW} / \mathrm{cm}^{2}$ set by the international guidelines as exposure limit above $6 \mathrm{GHz}$, see Table 2 in [7].

As far as SAR calculation is concerned, it is worth to underline that specific absorption rate (SAR) averaged over a $10 \mathrm{~g}$ cubic volume, considered by international guidelines for frequencies below $6 \mathrm{GHz}$, cannot be considered here, as the total mass of the samples (5g) is smaller than averaging mass considered by ICNIRP guidelines [6]. For this reason, we report the local SAR as numerically evaluated in each cell of the grid used to discretize the samples in the wells (point SAR). The computed SAR level averaged in each layer of the aqueous samples is shown in Figure 1C. The SAR distribution is non homogeneous in the different aqueous samples as larger values are reached in the central wells (2 and 5). This is due to near field condition exposure that entails a not local plane wave front of the field impinging on the samples. Moreover, as expected, top layers of the aqueous samples show larger SAR values than in depth layers, due to strong electromagnetic discontinuities and small penetration depth in salt water at $27 \mathrm{GHz},(0.65 \mathrm{~mm})$. Finally, the PLD also shows that power deposition into the central samples are about three times larger than that in the peripheral samples. However, as spermatozoa cells move randomly inside the sample, they will experience an average PLD values of about $22 \mathrm{~mW} / \mathrm{cm}^{3}$ in the outermost samples (well number 1,3, 4, 6) and about $52 \mathrm{~mW} / \mathrm{cm}^{3}$ in the innermost ones (well number 2 and 5), see Figure 1D. It is worth to stress that, although non homogeneous exposure are not suggested as good condition for dosimetric evaluation, in this case, as sperm vitality is observed on spermatozoa samples taken separately from each well, this may allow to understand is possible effects are associated to different power level conditions.

Last but not least, it is worth noticing that only numerical dosimetry has been performed because continuous thermal monitoring during exposure protocol is not viable due to invasiveness of most common temperature probes (thermocouple or optical fiber) at this working frequency. This notwithstanding, water temperature measurements of the 
aqueous sample reveal an increase of about $1^{\circ} \mathrm{C}$ after the exposure time with respect to the sham samples.

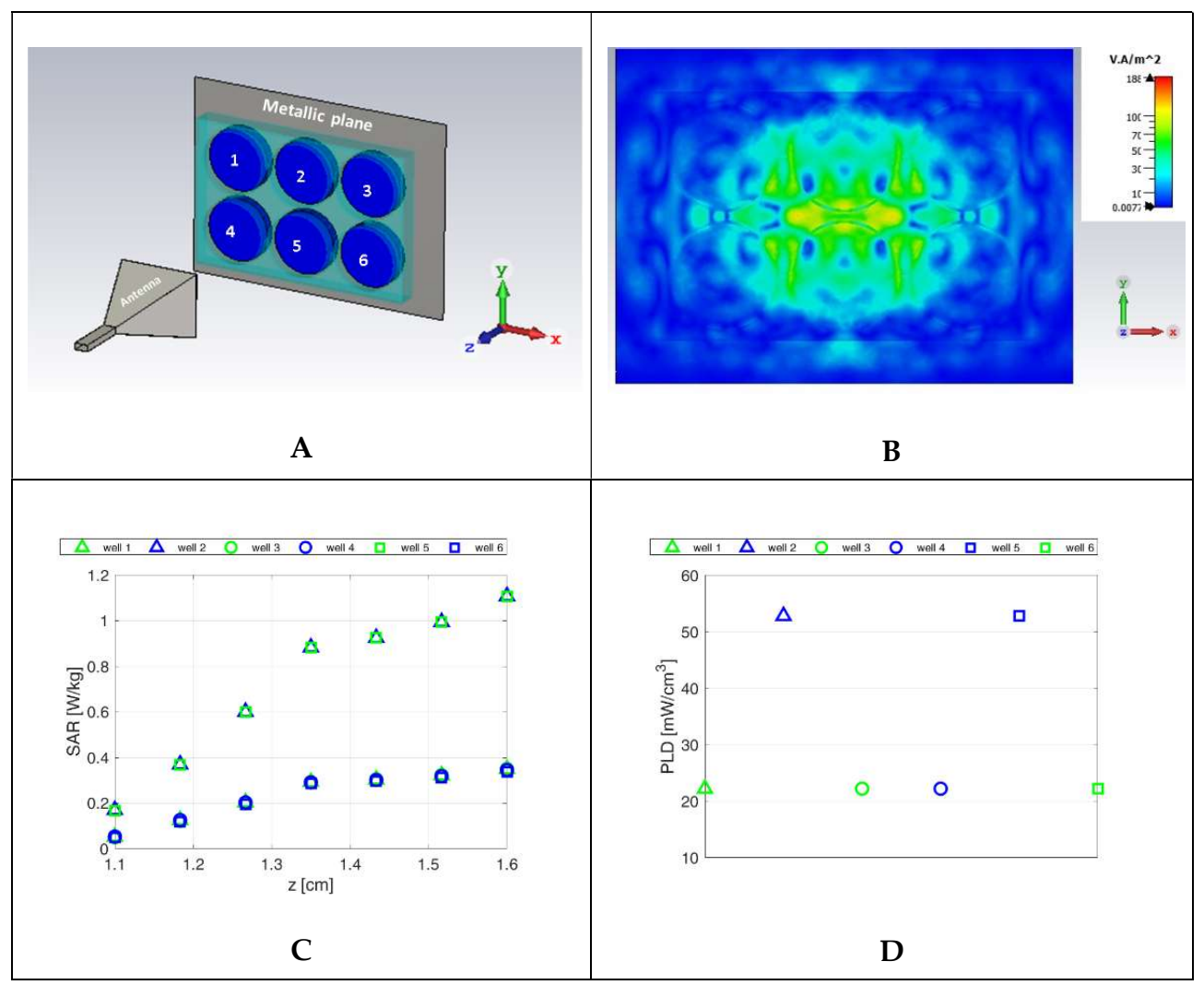

Figure 1. Experimental setup for numerical dosimetry. A) CAD model (6-well microplates numbered for understanding following figures). B) incident power density at distance 0.5 $\mathrm{cm}$ from the air-water interface of the aqueous samples. C) Average point SAR in each sample layer along depth z-direction. D) average PLD for each aqueous sample in the wells.

\subsection{Sperms vitality and motility analyses}

A significative decrement of sperm's vitality (Fig. 2) was observed already after 10 minutes of exposure at $27 \mathrm{GHz}(39 \%)$, compared to control samples. Eosin test showed a high mortality rate $(75 \%)$ of spermatozoa in all exposed samples already after 30 minutes (Fig. 3). We observed that electromagnetic fields irradiation induced also a significant decrease in sperm motility (Fig. 4) after 10 minutes of exposure (41\%).

Statistically significant differences $(p<0.001)$ have been obtained for all times of exposure between spermatozoa exposed and controls both for vitality and motility rate. 


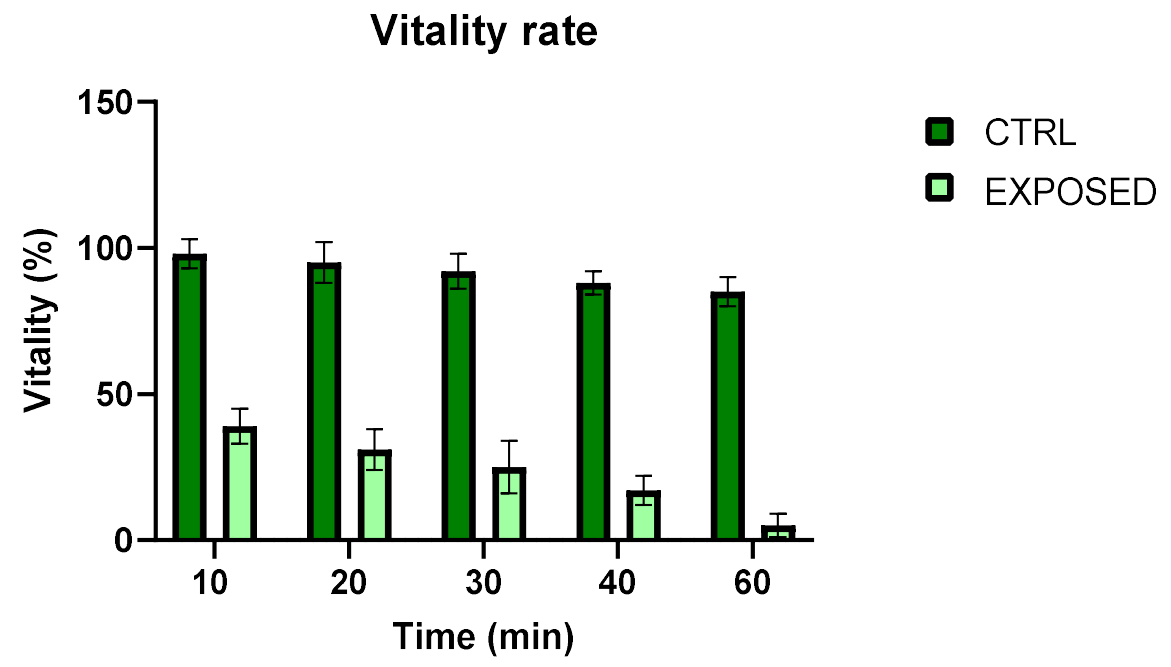

Figure 2. Vitality percentage at different times of exposure. The data represent the mean of observation performed in tenfold by same observer to avoid subjective differences in vitality evaluation.
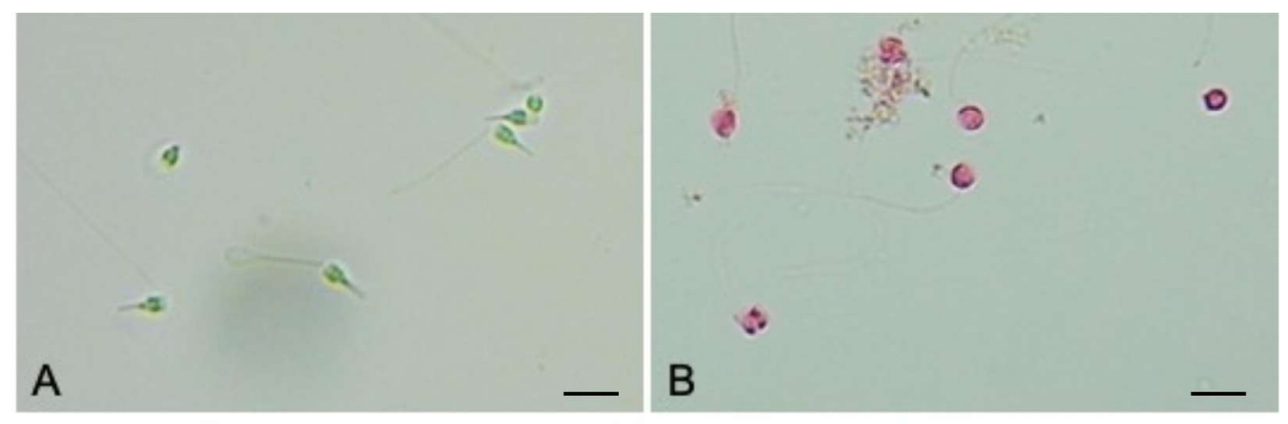

Figure 3. Vitality evaluation with Eosin Test on Mytilus galloprovincialis sperms. A) Untreated sample. B) After exposure to $27 \mathrm{GHz}$ for 30 minutes. Scale bar: $4 \mu \mathrm{m}$. 


\section{Motility rate}
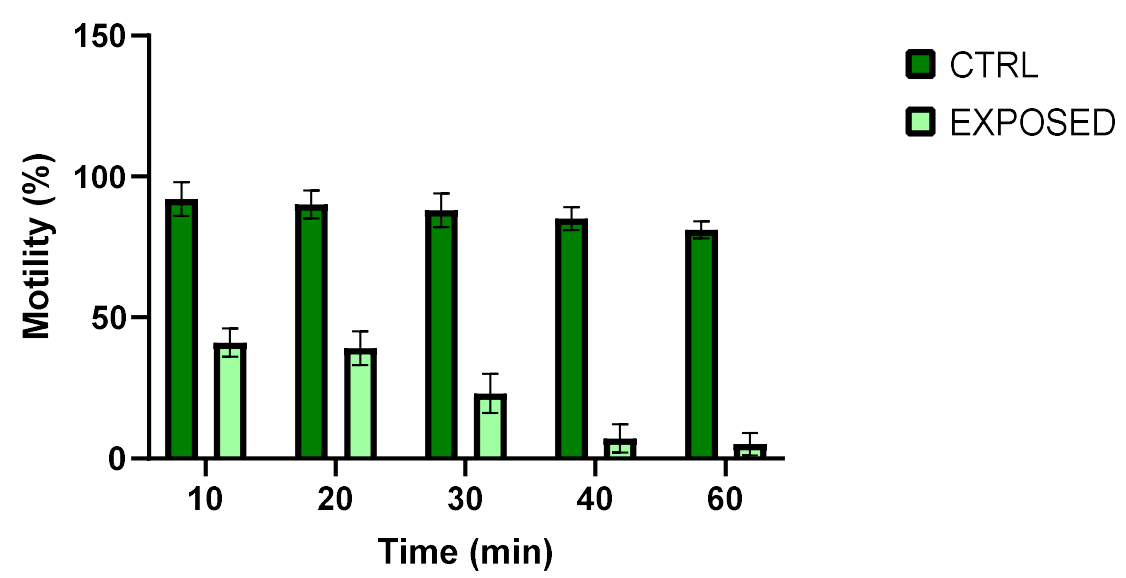

Figure 4. Motility percentage at different times of exposure. The data represent the mean of observation performed in tenfold by same observer to avoid subjective differences in motility evaluation.

\section{Discussion}

Fifth generation of global mobile communications will bring also a new era to maritime connectivity for real-time data transmissions. The development of mobile communication technologies has requested the assessment of possible risks occurring from exposures to radiofrequency electromagnetic fields. Whereas much is present in the literature on the effect on reproduction of electromagnetic fields in terrestrial animals model [17-22], little is known about the effect in aquatic animals, except for a few data concerning zebrafish $[16,17]$. Moreover, most of these effects were investigated at frequencies typical of previous cellular communications, mainly $2 \mathrm{G}$ and $3 \mathrm{G}$, i.e. frequencies much lower than $27 \mathrm{GHz}$. Recent evidences demonstrate that electromagnetic fields negatively affect sperm quality, sperm count, morphology and motility. In male rats 2 days old, exposed to EMF 1800 and $900 \mathrm{MHz}$ for 2 hours continuously per day for 90 days. The percentage of epididymal sperm motility was significantly higher in the $1800 \mathrm{MHz}$ group $(\mathrm{p}<0.05)$. The morphologically normal spermatozoa rates were higher and the tail abnormality and total percentage abnormalities were lower in the $900 \mathrm{MHz}$ group $(\mathrm{p}<0.05)$ [9]. In another study conducted always on rats exposed to $900 \mathrm{MHz}$ for 8 weeks, the Authors noticed a statistically significant decrease of epididymal sperm counts in the exposed group $(\mathrm{p}<0.001)$, a significant decrease of sperm motility and a significant $(p<0.001)$ increase in the frequency percentage of dead spermatozoa [18]. Guo et al. [23], exposing rats for 1 month to $220 \mathrm{MHz}$ demonstrated a decreased sperm count and survival rate of sperm $(\mathrm{p}<0.05)$, increased sperm abnormalities. No differences in body weight and development among the groups were found in mice of both sexes in rats exposed to $2.45 \mathrm{GHz}$ for $2 \mathrm{~h} /$ day, 5 days/week for 5 consecutive weeks, starting the day after birth [22]. On zebrafish exposed to $3.5 \mathrm{GHz}$ RFR, specific absorption rate (SAR) $\approx 8.27 \mathrm{~W} / \mathrm{Kg}$ from 6 hours post fertilization (hpf) to $48 \mathrm{hpf}$, have not been revealed significant impacts on mortality, morphology or photomotor response, but only a modest inhibition of startle response suggesting some levels of sensorimotor disruptions [23].

Several studies were conducted on evaluation of exposure by submarine power cables which produce both electric and magnetic fields. Although magnetic and electric fields' intensities decrease with distance away from the cable, marine invertebrate species are the major fauna exposed to, so they have received a greater attention [24]. A high-strength magnetic field applied during sea urchins (Lytechinus pictus and Strongylocentrotus purpuratus) fertilization delayed cell division in embryos $[25,26]$. Moreover, Levin and Ernst 
[25] highlighted an increase in developmental abnormalities, but only in L. pictus. However, a 93-day exposure throughout the reproductive period of the blue mussel (Mytilus edulis) did not affect either its condition index or its gonad development index [27].

In our study, a notable decrease in the vitality of M. galloprovincialis spermatozoa after only 10 minutes of exposure at $27 \mathrm{GHz}$ was shown. We observed also that electromagnetic fields irradiation induced a significant decrease in sperm motility after 10 minutes of exposure. If confirmed, possible explanation of our observation is related to a direct action of the electromagnetic field on phospholipid bilayer of cells membrane. This effect had been investigated, but for at higher frequencies (around $60 \mathrm{GHz}$ ), on liposomes and on giant unilamellar vesicles [28-30].

\section{Conclusions}

The study indicates that electromagnetic fields at $27 \mathrm{GHz}$ can affect the sperm quality in marine mussel Mytilus galloprovincialis. The significative decrease observed in sperm motility after only 10 minutes of exposure represents a crucial factor need to be considered because it can threat reproductivity of the species. This study provides useful data on potential impact of high frequency EMFs on aquatic animals and cells, that currently are poorly investigated. Future research could benefit from specific investigation on the impact of $5 \mathrm{G}$ to better monitoring effects on animal organisms and to fill the gap currently known about the interactions with artificial sources of electromagnetic fields.

Author Contributions: Conceptualization, L.D.D. and M.V.B.; methodology, R.P., E.M.S, M.C., S.C.P. and G.S.; investigation, C.S. and S.I.; data curation, S.I., C.S., S.C.P. and G.S.; writing-original draft preparation, L.D.D. and M.V.B.; writing-review and editing, R.P., E.M.S., A.S. and M.V.B.; supervision, L.D.D., R.P. and M.V.B.; funding acquisition, L.D.D. and M.V.B. All authors have read and agreed to the published version of the manuscript.

Funding: This research has been funded by “Programma ricerca di Ateneo UNICT 2020-22 linea 2".

Conflicts of Interest: The authors declare no conflict of interest.

\section{References}

1. Attaran, $\mathrm{M}$. The impact of $5 \mathrm{G}$ on the evolution of intelligent automation and industry digitization. J. Ambient. Intell. Human Comput. 2021, 1-17.

2. Foster, K.R.; Vijayalaxmi. Needed: More Reliable Bioeffects Studies at "High Band” 5G Frequencies. Front. Comms. Net. 2021, 2,721925 .

3. Hardell, L.; Carlberg, M. Health risks from radiofrequency radiation, including 5G, should be assessed by experts with no conflicts of interest. Oncol Lett. 2020, 20(4), 15.

4. Simkó, M.; Mattsson, M.O. 5G Wireless Communication and Health Effects-A Pragmatic Review Based on Available Studies Regarding 6 to $100 \mathrm{GHz}$. Int. J. Environ. Res. Public Health. 2019, 16(18), 3406.

5. Mattsson, M.O.; Simkó, M.; Foster, K.R. 5G New Radio Requires the Best Possible Risk Assessment Studies: Perspective and Recommended Guidelines. Front. Comms. Net. 2021, 2, 724772.

6. Agence nationale de sécurité sanitaire de l'alimentationde l'environnement et du travail (ANSES) Expositions aux champs électromagnétiques liées au déploiement de la technologie de communication « 5G » et effets sanitaires éventuels associés. Rapport d'expertise collective, 2021.

7. International Commission on Non-Ionizing Radiation Protection (ICNIRP), "Guidelines for limiting exposure to time-varying electric, magnetic and electromagnetic fields (up to $300 \mathrm{GHz}$ )". Health Phys. 1998, 74(4), 494-522.

8. Dreujou, E.; Desroy, N.; Carrière, J.; Tréau de Coeli, L.; McKindsey, C.W.; Archambault, P. Determining the Ecological Status of Benthic Coastal Communities: A Case in an Anthropized Sub-Arctic Area. Front. Mar. Sci. 2021, 8, 637546.

9. Klimley, A.; Wyman, M.; Kavet, R. Assessment of Potential Impact of Electromagnetic Fields from Undersea Cable on Migratory Fish Behaviour (No. FINAL REPORT, DOE-EPRI-Ee0006382 OCS Study BOEM 2016-041). 2016, Electric Power Research Institute (EPRI), Palo Alto, CA, (USA).

10. Ohman, M.; Sigray, P.; Westerberg, H. Offshore windmills and the effects of electromagnetic fields on fish. Ambio. 2007, 36 (8), 630-633. 
11. Tricas, T.; Gill, A. Effects of EMFs from Undersea Power Cables on Elasmobranchs and Other Marine Species (No. OCS Study BOEMRE 2011-09). 2011, U.S. Department of the Interior, Bureau of Ocean Energy Management (BOEM), Camarillo, CA (USA).

12. Wildt, D.E.; Comizzoli, P.; Pukazhenthi, B.; Songsasen, N. Lessons from biodiversity - the value of nontraditional species to advance reproductive science, conservation, and human health. Mol. Reprod. Dev. 2010, 77(5), 397-409.

13. Boncel, S.; Kyzioł-Komosińska, J.; Krzyżewska, I.; Czupioł, J. Interactions of carbon nanotubes with aqueous/aquatic media containing organic/inorganic contaminants and selected organisms of aquatic ecosystems - A review. Chemosphere. 2015, 136, 211-221.

14. Maresca, V.; Fusaro, L.; Sorbo, S.; Siciliano, A.; Loppi, S.; Paoli, L., Monaci, F.; Asadi karma, E.; Piscopo, M.; Guida, M.; Galdiero, E.; Insolvibile, M.; Basile, A. Functional and structural biomarkers to monitor heavy metal pollution of one of the most contaminated freshwater sites in Southern Europe. Ecotoxicol. Environ. Saf. 2018, 163, 665-673.

15. Piscopo, M.; Notariale, R.; Rabbito, D.; Ausi, J.; Olanrewaju, O.S.; Guerriero, G. Mytilus galloprovincialis (Lamarck, 1819) spermatozoa: Hsp70 expression and protamine-like protein property studies. Environ. Sci. Pollut. Res. 2018, 25(13), 1295712966.

16. Reitsema, R.E., Meire, P.; Schoelynck, J. The future of freshwater macrophytes in a changing world: Dissolved organic carbon quantity and quality and its interactions with macrophytes. Front. Plant Sci. 2018, 9.

17. Ozlem Nisbet, H.; Nisbet, C.; Akar, A.; Cevik, M.; Karayigit, M.O. Effects of exposure to electromagnetic field (1.8/0.9 GHz) on testicular function and structure in growing rats. Res. Vet. Sci. 2012, 93, 1001-5.

18. Bin-Meferij, M.M.; El-Kott, O.F. The neuroprotective effects of Moringa oleifera against mobile phone electromagnetic radiation-induced infertility in rats. Int. J. Clin. Exp. Med. 2015, 8, 12487-97.

19. Bilgici, B.; Gun, S.; Avci, B.; Akar, A.; Engiz, B. What is adverse effect of wireless local area network, using $2.45 \mathrm{GHz}$, on the reproductive system? Int. J. Radiat. Biol. 2018, 94, 1054-1061.

20. Saygin, M.; Asci, H.; Ozmen, O.; Cankara F.N.; Dincoglu D.; Ilhan I. Impact of 2.45 GHz microwave radiation on the testicular inflammatory pathway biomarkers in young rats: The role of gallic acid. Environ. Toxicol. 2016, 31, 1771-1784.

21. Ozguner, M.; Koyu, A.; Cesur, G.; Ural, M.; Ozguner, F.; Gokcimen, A.; Delibas, N. Biological and morphological effects on the reproductive organ of rats after exposure to electromagnetic field. Saudi Medical Journal. 2005, 26, 405-410.

22. Sambucci, M.; Laudisi, F.; Nasta, F.; Pinto, R.; Lodato, R.; Lopresto, V.; Altavista, P.; Marino, C.; Pioli, C. Early life exposure to $2.45 \mathrm{GHz}$ WiFi-like signals: effects on development and maturation of the immune system. Prog. Biophys. Mol. Biol. 2011, 107, 393-8.

23. Guo, L.; Lin, J.J.; Xue, Y.Z.; An, G.Z.; Zhang, J.P.; Zhang, K.Y.; He, W.; Wang, H.; Li, W.; Ding, G.R. Effects of 220 MHz pulsed modulated radiofrequency field on the sperm quality in rats. Int. J. Environ. Res. Public Health. 2019, 16, 1286.

24. Albert, L.; Deschamps, F.; Jolivet, A.; Olivier, F.; Chauvaud, L.; Chauvaud, S. A current synthesis on the effects of electric and magnetic fields emitted by submarine power cables on invertebrates. Mar. Environ. Res. 2020, 159, 104958.

25. Levin, M.; Ernst, S. Applied DC magnetic fields cause alterations in the time of cell divisions and developmental abnormalities in early sea-urchin embryos. Bioelectromagnetics 1997, 18 (3), 255-263.

26. Zimmerman, S.; Zimmerman, A.; Winters, W.; Cameron, I. Influence of 60-Hz magnetic fields on sea urchin development. Bioelectromagnetics 1990, 11 (1), 37-45.

27. Bochert, R.; Zettler, M. Long-term exposure of several marine benthic animals to static magnetic fields. Bioelectromagnetics 2004, 25 (7), 498-502.

28. D’ Agostino, S.; Della Monica, C.; Palizzi, E., Di Pietrantonio, F.; Benetti, M.; Cannatà, D.; Cavagnaro, M., Sardari, D., Stano, P., Ramundo-Orlando, A. Extremely high frequency electromagnetic fields facilitate electrical signal propagation by increasing transmembrane potassium efflux in an artificial axon model. Scientific reports, 2018, 8(1), 1-10.

29. Cosentino, K.; Beneduci, A.; Ramundo-Orlando, A.; Chidichimo, G. The influence of millimeter waves on the physical properties of large and giant unilamellar vesicles. Journal of biological physics, 2013, 39(3), 395-410.

30. Di Donato, L; Cataldo, M.; Stano, P.; Massa, R.; Ramundo-Orlando, A. Permeability changes of cationic liposomes loaded with carbonic anhydrase induced by millimeter waves radiation. Radiation research, 2012, 178(5), 437-446. 